\title{
Urinary excretion half life of trichloroacetic acid as a biomarker of exposure to chlorinated drinking water disinfection by-products
}

\author{
E L Bader, S E Hrudey, K L Froese
}

Occup Environ Med 2004;61:715-716. doi: 10.1136/oem.2003.008698

\begin{abstract}
Aims: To measure accurately urinary elimination half life of trichloroacetic acid (TCAA).

Methods: A longitudinal pilot exposure/intervention study measured the elimination half life of TCAA in urine. Beverage consumption was limited to a public water supply and bottled water of known TCAA concentration, and ingestion volume was managed. The five participants limited fluid consumption to only the water provided. Consumption journals were kept by each participant and their daily first morning urine (FMU) samples were analysed for TCAA and creatinine. TCAA elimination half life curves were generated from a two week washout period using TCAA-free bottled water.

Results: Individual elimination half lives ranged from 2.1 to 6.3 days, for single compartment exponential decay, the model which fit the data.

Conclusion: Urinary TCAA is persistent enough to be viable as a biomarker of medium term (days) exposure to drinking water TCAA ingestion within a range of realistic concentrations.
\end{abstract}

C hlorinated disinfection by-products (DBPs) in drinking water pose a challenge to the epidemiological study of both cancer and adverse reproductive outcomes. ${ }^{1}$ Reviews of evidence on adverse reproductive outcomes have consistently cited a major need to improve individual exposure assessment. ${ }^{1-3}$ Biomarkers have been suggested as the potential "gold standard" for achieving this improvement," and their development and validation has been recommended. ${ }^{1}$

Of the many DBPs that are present in chlorinated waters, the trihalomethanes (THMs) and haloacetic acids (HAAs) are the major DBPs. Consequently, these classes of DBPs have received the most attention as potential biomarkers of exposure for DBPs. Initial work by Weisel and co-workers ${ }^{4}$ and limited pharmacokinetic literature ${ }^{5}$ showed promise for trichloroacetic acid (TCAA) for integrating medium term exposure to drinking water DBPs. We previously performed a longitudinal pilot study to measure the urinary elimination half life of TCAA. ${ }^{7}$ Limitations in that study design indicated the need for a better controlled study to provide greater confidence in the human TCAA elimination kinetics.

\section{METHODS}

\section{Volunteer recruitment}

Volunteers from Edmonton, herein referred to as City A, were sought for participation in the study. Five volunteers in total, three males (ages 27, 37, 52) and two females (ages 28, 29), were recruited. All of the volunteers were either students or staff from our research group. The Health Ethics Research Board of the University of Alberta approved the study protocol, and we obtained informed written consent from participants at enrolment in the trial.

\section{Study design}

Drinking water in City A had very low levels of TCAA (5$20 \mu \mathrm{g} / \mathrm{l})$; therefore, water was imported from another city (referred to as City B) with higher TCAA (50-180 $\mu \mathrm{g} / \mathrm{l})$.

This study was designed to measure the half life of urinary elimination for TCAA. Participants consumed normal tap water from City B for two weeks and then switched to TCAAfree bottled water for two weeks. Bottled water was obtained from the Sierra Spring Water Company, and was confirmed TCAA-free in our laboratory. FMU samples were collected from each volunteer for every day of the study.

Each volunteer received instructions on how to collect an FMU sample and how to fill out the daily diary. The participants recorded the collection times of the urine samples, previous urination times, the time and amount of all fluid consumption, the time and amount of any medication taken, and the time and duration of water related activities.

\section{Sampling and laboratory analysis}

Entire urine voids were collected in $1000 \mathrm{ml}$ high density polyethylene bottles. All samples were extracted within 24 hours of collection. TCAA concentrations in the urine samples were measured according to a modified version of US EPA Method $552.2^{8}$ that employed the use of solid phase microextraction (SPME).

\section{Urinary elimination half life estimation}

Urinary elimination half lives were estimated in two ways. The first used the TCAA concentration measured in FMU samples divided by urinary creatinine concentrations. The TCAA value for day zero was the average of approximately 14 days consuming City B tap water, with day 1 the first day of the bottled water intervention. The logarithm of the creatinine normalised TCAA urinary excretion was evaluated against time. Data were confirmed to fit a single compartment, first order model using pharmacokinetic software (GraphPad Prism, version 3).

The second method used TCAA excretion rate as an alternative to creatinine normalised concentrations. This TCAA elimination rate was calculated using the time difference between last reported urination and the FMU. The logarithm of this rate was evaluated against time. Otherwise, half lives were estimated in the same manner.

\section{RESULTS}

The TCAA urinary elimination half lives obtained for the five participants using creatinine correction were: 2.1 days $\left(R^{2}=0.83\right), 2.3$ days $\left(R^{2}=0.94\right), 2.5$ days $\left(R^{2}=0.88\right), 5.0$

Abbreviations: DBPs, drinking water chlorinated disinfection byproducts; FMU, first morning urine void; HAA, halogenated (chloro- and bromo-) acetic acid; SPME, solid phase microextraction; TCAA, trichloroacetic acid; THM, trihalomethanes 


\section{Main messages}

- Urinary trichloroacetic acid is persistent enough to be viable as a biomarker for drinking water exposure to this non-volatile disinfection by-product.

- The urinary excretion half life of trichloroacetic acid is long enough to integrate drinking water exposure over several days.

days $\left(R^{2}=0.80\right)$, and 6.3 days $\left(R^{2}=0.76\right)$. Figure 1 shows the urinary elimination curve for one participant, with the creatinine adjusted method. Similar, but slightly inferior (in terms of $\mathrm{R}^{2}$ values) results were obtained using the urinary elimination rate method: 2.2 days $\left(R^{2}=0.72\right), 2.2$ days $\left(\mathrm{R}^{2}=0.94\right), 2.2$ days $\left(\mathrm{R}^{2}=0.70\right), 4.6$ days $\left(\mathrm{R}^{2}=0.84\right)$, and 5.3 days $\left(R^{2}=0.62\right)$.

\section{DISCUSSION}

There is limited literature documenting the pharmacokinetics of human TCAA elimination when TCAA is administered directly because most literature examines the behaviour of TCAA as a metabolite of either chloral hydrate or trichloroethylene. Published half life values for TCAA when administered directly as the parent compound (as sodium trichloroacetate) were for substantially higher doses of TCAA $(\geqslant 10000$ times) than occur in drinking water ingestion exposure, raising concerns for how well these data will characterise the latter. Three volunteers showed elimination half life values of 3.1, 3.2, and 4.1 days when given relatively massive intravenous doses of sodium trichloroacetate: 28.1, 60.2 , and $37.3 \mathrm{mg} / \mathrm{kg}$, respectively. ${ }^{10}$

The only urinary elimination half life values previously published for TCAA ingested from drinking water were 2.3 days $\left(R^{2}=0.74\right), 2.9$ days $\left(R^{2}=0.70\right)$, and 3.7 days $\left(R^{2}=0.45\right)$, based on our study with only seven elimination curve data points per participant. ${ }^{7}$ The high quality fit $\left(\mathrm{R}^{2}\right.$ from 0.76 to 0.94 among five subjects) to the one compartment, exponential elimination model for our current study validates the earlier promising findings of a medium duration urinary elimination half life for TCAA.

The range of TCAA urinary excretion half life values determined in this study (2.1-6.3 days) confirms that the urinary elimination half life of TCAA is significantly greater than the time interval between successive drinking water

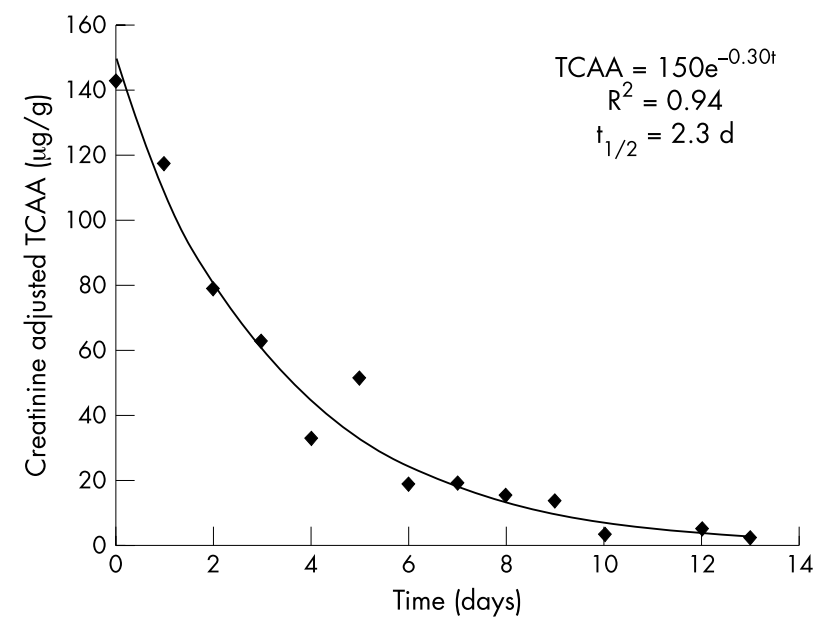

Figure 1 TCAA urinary elimination half life estimation using creatinine adjusted TCAA concentration in FMU.

\section{Policy implications}

- Epidemiological studies of disinfection by-products need improved, hypothesis testing studies to improve their contribution to causal inference.

- Future epidemiological studies should explore the utility of urinary trichloroacetic acid as a biomarker of individual drinking water ingestion of chlorinated disinfection by-products.

exposures to TCAA given periodic daily consumption. TCAA will accumulate in plasma towards a quasi-steady state concentration, so the urinary excretion of TCAA will reflect average exposure over several days. TCAA appears to be persistent enough to provide a valid exposure biomarker for ingested TCAA from chlorinated drinking water for an exposure window of several days.

\section{ACKNOWLEDGEMENTS}

The authors thank the cooperative participants, and Michael Ongley for laboratory assistance.

\section{Authors' affiliations}

E L Bader, S E Hrudey, K L Froese, Department of Public Health Sciences, University of Alberta, 10-102 Clinical Sciences Bldg, Edmonton, AB T6G 2G3, Canada

Financial support was from: the Natural Sciences and Engineering Research Council-a postgraduate scholarship to Erin Bader and Strategic Grant No. 215854-98; and Alberta Heritage Foundation for Medical Research and Alberta Health and Wellness.

Correspondence to: Prof. S E Hrudey, Department of Public Health Sciences, University of Alberta, 10-102 Clinical Sciences Bldg, Edmonton, AB T6G 2G3, Canada; steve.hrudey@ualberta.ca

Accepted 2 November 2003

\section{REFERENCES}

1 Arbuckle TE, Hrudey SE, Krasner SW, et al. Assessing exposure in epidemiologic studies to disinfection by-products in drinking water: report from an international workshop. Environ Health Perspect 2002;110:53-60.

2 Graves CG, Matanoski GM, Tardiff RG. Weight of evidence for an association between adverse reproductive and developmental effects and exposure to disinfection by-products: a critical review. Regul Toxicol Pharmacol 2001;34:103-24.

3 Swan SH, Waller K. Disinfection by-products and adverse pregnancy outcomes: what is the agent and how should it be measured? Epidemiology 1998;9:479-81.

4 Weisel $\mathrm{CP}, \mathrm{Kim} \mathrm{H}$, Haltmeier $\mathrm{P}$, et al. Exposure estimates to disinfection byproducts of chlorinated drinking water. Environ Health Perspect 1999; 107:103-10.

5 Breimer DD, Ketelaars HCJ, Van Rossum JM. Gas chromatographic determination of chloral hydrate, trichloroethanol, and trichloroacetic acid in blood and urine employing head-space analysis. J Chromatogr 1974;99:55-63.

6 Humbert L, Jacquemont MC, Leroy E, et al. Determination of chloral hydrate and its metabolites (trichloroethanol and trichloroacetic acid) in human plasma and urine using electron capture gas chromatography. Biomed Chromatogr 1994;8:273-7.

7 Froese KL, Sinclair M, Hrudey SE. Trichloroacetic acid as a biomarker of exposure to disinfection by-products in drinking water: a human exposure trial in Adelaide, Australia. Environ Health Perspect 2002;1 10:679-87.

8 US Environmental Protection Agency. Method 552.2, Revision 1.0. Determination of haloacetic acids and dalapon in drinking water by liquidliquid extraction, derivatization and gas chromatography with electron capture detection Report No: EPA/600/R-95/131. Cincinnati, OH: US Environmental. Protection Agency, Office of Research and Development, National Exposure Research Laboratory, 1995.

9 Sarrion MN, Santos FJ, Galceran MT. Solid-phase microextraction coupled with gas chromatography-ion trap mass spectrometry for the analysis of haloacetic acids in water. J Chromatogr A 1999;859:159-71.

10 Paykoc ZV, Powell JF. The excretion of sodium trichloroacetate. J Pharmacol Exp Ther 1945;85:289-93. 